\title{
STC1 regulates glioblastoma migration and invasion via the TGF- $\beta$ /SMAD4 signaling pathway
}

\author{
YAN XIONG $^{1}$ and QIBAI WANG ${ }^{2}$ \\ ${ }^{1}$ Department of Neurosurgery, Chongqing Ninth People's Hospital, Chongqing 400715; ${ }^{2}$ Department of Neurosurgery, \\ Chongqing Red Cross Hospital (People's Hospital of Jiangbei District), Chongqing 400020, P.R. China
}

Received October 22, 2018; Accepted May 1, 2019

DOI: $10.3892 / \mathrm{mmr} .2019 .10579$

\begin{abstract}
Stanniocalcin-1 (STC1) is involved in cancer progression; however, the function of STC1 in glioblastoma remains unknown. In the present study, the expression levels of STC1 protein in glioblastoma were detected using immunohistochemistry. The expression levels of STC1, SMAD2/3 and SMAD4 proteins, following silencing of STC1, were assessed via western blotting. EdU and Transwell assays were performed to determine the proliferation and migration ability of the cells. The mRNA expression levels of STC1, SMAD4 and microRNA (miR)-34a were determined using quantitative PCR. The expression levels of STC1 were increased in glioblastoma tissues. STC1 revealed a significant association with poor outcome in patients with glioblastoma $(\mathrm{P}<0.05)$. The proliferation and invasion abilities were repressed in LN229 cells infected with LV3-shSTC1-1 and LV3-shSTC1-2 compared with LV3-NC. By contrast, the proliferation and invasion abilities were increased in T98G cells infected with LV5-STC1 compared with LV5-NC $(\mathrm{P}<0.05)$. The expression levels of STC1, SMAD2/3 and SMAD4 were decreased in LN229 cells infected with LV3-shSTC1-1 and LV3-shSTC1-2 compared with LV3-NC. However, the expression levels of STC1, SMAD2/3 and SMAD4 were elevated in T98G cells infected with LV5-STC1 compared with LV5-NC. The expression levels of miR-34a were decreased following silencing of STC1 $(\mathrm{P}<0.05)$. The expression levels of SMAD4 were decreased when transfected with miR-34a mimics $(\mathrm{P}<0.05)$. The luciferase activity of the wild-type 3'untranslated region of SMAD4 was decreased following transfection with miR-34a mimics $(\mathrm{P}<0.05)$. Silencing of STC1 inhibited the growth of LN229 in vivo. In conclusion, STC1 expression levels were increased in the present
\end{abstract}

Correspondence to: Dr Qibai Wang, Department of Neurosurgery, Chongqing Red Cross Hospital (People's Hospital of Jiangbei District), 40 Road Gate, Yuzhong, Chongqing 400020, P.R. China

E-mail: wangqibosci@163.com

Key words: SMAD2/3, stanniocalcin-1, SMAD4, microRNA-34a, glioblastoma study, and it was revealed that STC1 regulated glioblastoma malignancy. This phenotype was observed in the SMAD2/3 and SMAD4 pathways.

\section{Introduction}

Glioblastoma is one of the most common types of cancer and accounts for $46.1 \%$ of all glioma cases in 2015, with an incidence of $\sim 3.20$ per 100,000 individuals every year (1-3). In recent years, surgery and adjuvant chemotherapy have made great progress alongside the development of medical technology; however, the 5-year survival rate of patients with glioblastoma remains poor. The main cause of this poor prognosis is tumor invasion and metastasis (4-6), though the underlying molecular mechanism remains unclear; therefore, elucidating this molecular mechanism has important clinical significance.

Stanniocalcin-1 (STC1) is a secreted glycoprotein hormone (7). It maintains calcium homeostasis in teleost fish $(8,9)$. Some studies have reported that STC1 is expressed in human tissues, including the ovary, kidney, brain, lung, heart, muscle and skeletal tissues (10-14). In addition, STC1 has been demonstrated to be associated with angiogenesis, apoptosis and inflammation $(15,16)$. Accumulating evidence has demonstrated that STC1 plays a crucial role in numerous different types of cancer. Aberrant expression of STC1 has been observed in human carcinoma samples, including those from colorectal cancer, hepatocellular carcinoma, breast cancer, lung adenocarcinoma and thyroid carcinomas $(13,17-19)$. In liver cancer, high expression levels of STC1 result in decreased energy metabolism and, in turn, stimulate the AMPK pathway and inhibit p70S6K/p-rpS6 signaling (20). In cervical cancer, STC1 regulates cell apoptosis via NF- $\kappa$ B phospho-P65 Ser536 (21). Another study demonstrated that the elevated expression levels of STC-1 promoted the invasion of triple negative breast cancer cells through the activation of the c-Jun N-terminal kinase (JNK)/c-Jun signaling pathway (22). However, the expression and function of STC1 has not yet been elucidated in glioblastoma.

In the present study, the expression level of STC1 was examined in glioblastoma tissues and cell lines. Additionally, the function and the mechanisms underlying STC1 were investigated in this type of cancer. 


\section{Materials and methods}

Tissues. The patients were admitted to Chongqing Red Cross Hospital between January 2014 and January 2017 and diagnosed with glioblastoma by a pathologist. All patients underwent lesion resection and all patients with glioma received a postoperative pathological diagnosis. The present study was approved by The Ethics Committee of Chongqing Red Cross Hospital, and written informed consent was obtained from all patients. Samples from 80 cases of glioblastoma and adjacent non-cancerous tissue were obtained. The distance between the glioblastoma and normal tissue was $2 \mathrm{~cm}$. The patients included 38 women and 42 men, with an age range of 16-62 years. Patients with glioblastoma had their diagnosis confirmed by pathological examination. Normal brain tissue was derived from surgical treatment of benign brain lesions, excluding patients with inflammation and malignant tumors.

Immunohistochemistry (IHC) staining. The expression of STC1 in glioblastoma was detected via IHC staining as previously described (23). Tissue were fixed with $10 \%$ formalin at room temperature for $48 \mathrm{~h}$, paraffin-embedded (thickness, $4 \mu \mathrm{m}$ ) tissue sections were deparaffinized at room temperature for $1 \mathrm{~h}$, washed with xylene at room temperature for $15 \mathrm{~min}$ and rehydrated in a descending alcohol series for $20 \mathrm{~min}$. Following antigen retrieval and inactivation of endogenous peroxidase, the sections were blocked with $10 \%$ goat serum (OriGene Technologies, Inc.) at $37^{\circ} \mathrm{C}$ for $15 \mathrm{~min}$ and incubated with anti-STC1 primary antibody at $37^{\circ} \mathrm{C}$ for $2 \mathrm{~h}$ (1:200; catalog no. ab229477; Abcam), followed by incubation with secondary antibody anti-rabbit immunoglobulin $\mathrm{G}$ conjugated to horseradish peroxidase (HRP; cat. no. SP-9000; ZSBio; OriGene Technologies, Inc.) for $1 \mathrm{~h}$ at room temperature, the secondary antibody was provided in the kit and no additional dilution was required (cat. no. SP-9000; ZSBio; OriGene Technologies, Inc.). After washing with PBS, the sections were incubated in horseradish enzyme-labeled chain avidin solution for $15 \mathrm{~min}$ at $37^{\circ} \mathrm{C}$ and washed again. Subsequently, the proteins were visualized using 3,3'-diaminobenzidine (cat. no. ZLI-9017; OriGene Technologies, Inc.). Sections were observed with a light microscope (magnification, x20). The STC1-positive cells were scored as follows: 0 (negative intensity), 1 (weak intensity), 2 (moderate intensity) or 3 (strong intensity). The percentage of STC1-positive cells was scored as follows: 0, $1,2,3$ and 4 from no cytoplasm expression, and 1-25, 26-50, 51-75, and 76-100\% cytoplasm expression, respectively (23). ImagePro Plus 6.0 (Media Cybernetics, Inc.) was used to analyze the stained sections. The final STC1 staining score was obtained by multiplying the intensity and percentage score. The low expression was defined as staining score $\leq 6$, while the high expression was defined as staining score $>6$.

Cell culture and transfection. The U251 cell line was purchased from the Chinese Academy of Sciences Cell Library (3131C0001000700058). LN229 [American Type Culture Collection (ATCC ${ }^{\circledR}$ ) CRL-2611] and T98G (ATCC ${ }^{\circledR}$ CRL-1690 $\left.{ }^{\mathrm{TM}}\right)$ were purchased from the ATCC. The U87 cell line (Chinese Academy of Sciences Cell Library) represents glioblastoma of unknown origin. U251 cells were purchased form The Chinese Academy of Sciences
Cell Library. The human astrocyte cell lines LN229 and T98G were purchased from ATCC. The four glioma cell lines were cultured in RPM1 1640 medium supplemented with $1 \%$ penicillin/streptomycin and 10\% FBS (Gibco; Thermo Fisher Scientific, Inc.) at $37^{\circ} \mathrm{C}$ and $5 \% \mathrm{CO}_{2}$. Small interfering (si)RNAs, miRNA mimics and inhibitor were purchased from Shanghai GenePharma Co., Ltd. After 48 h, cells were collected for subsequent experimentation. The sequences of miR-34a-5p inhibitor, miR-34a-5p mimic and negative control were used as follows: miR-34a-5p inhibitor, 5'-ACAACCAGCUAAGACACUGCCA-3'; miR-34a-5p mimics, 5'-UGGCAGUGUCUUAGCUGGUUGU-3'; negative control, 5'-UUCUCCGAACGUGUCACGUTT-3'. The concentration of miRNA transfected was $5 \mathrm{nM}$. siRNAs were obtained from Shanghai GenePharma Co., Ltd. The siRNA sequences were as follows: STC1-1, 5'-GCAGCAGCAUCACCAGCAACA-3'; STC1-2, 5'-GAUCCACAUCUUCACUCAAGC-3'; negative control siRNA, 5'-UUCUUCGAAGGUGUCACGU-3'. Lentiviral vectors expressing shRNA-targeting STC1 (named LV3-shSTC1-1 and LV3-shSTC1-2) and STC1-lentiviral expression vector (named LV5-STC1) were obtained from Genepharm, Inc. The sequences of the shRNA were the same as the siRNA sequences. In total, $10 \mathrm{nM}$ siRNA was transfected into the cells. Cells were transfected using the siRNA-Mate transfection reagent (Genepharm, Inc.).

Luciferase reporter assay. Cells were transfected in six-well plates with $2 \mu \mathrm{g}$ of a PReport plasmid (Genepharm, Inc.) containing the STC1 3'UTR including the binding site of miR-34a. Cells in each well were cotransfected using Lipofectamine 2000 (Invitrogen; Thermo Fisher Scientific, Inc.) with $20 \mathrm{nM}$ miR-34 mimics or negative control mimics. After $48 \mathrm{~h}$, luciferase assays were performed using a dual-luciferase reporter assay system (Promega Corporation). Luciferase activity was normalized relative to Renilla luciferase activity.

Total RNA isolation and reverse transcription-quantitative $(R T-q) P C R$. Total RNA was extracted from the cancer cells using RNA pure High-purity Total RNA Rapid Extraction kit (BioTeke Corporation; cat. no. RP1201) according to the manufacturer's protocol. RT and qPCR kits (both from Bio-Rad Laboratories, Inc.) were used to determine the mRNA expression levels. The RT and qPCR reactions were performed as previously described (18). The RT reaction protocol was performed using three sequential incubations of 5 at $25^{\circ} \mathrm{C}, 30 \mathrm{~min}$ at $42^{\circ} \mathrm{C}$ and $5 \mathrm{~min}$ at $85^{\circ} \mathrm{C}$. The thermocycling conditions were as follows: Denaturation at $95^{\circ} \mathrm{C}$ for $10 \mathrm{sec}, 60^{\circ} \mathrm{C}$ for $20 \mathrm{sec}, 72^{\circ} \mathrm{C}$ for $10 \mathrm{sec}$. qPCR kit (All-in-One qPCR Mix SYBR Green) and primers were purchased from GeneCopoeia, Inc. The relative mRNA expression levels were calculated using the comparative quantification cycle $2^{-\Delta \Delta \mathrm{Cq}}$ method (18). GAPDH was used as an endogenous control to normalize the expression of the target genes. The primers for STC1 (cat. no. HQP017782) and GAPDH (cat. no. HQP064347) were purchased from GeneCopoeia, Inc.

Western blot analysis. The expression of STC1, p-SMAD2/3, p-SMAD4 and GAPDH proteins was determined via western blotting as previously described (19-21), using STC1 
A

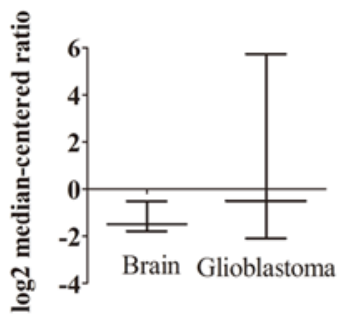

$\mathrm{D}$

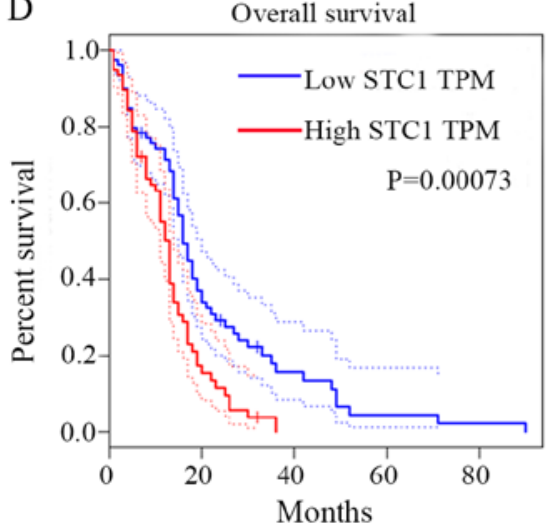

B

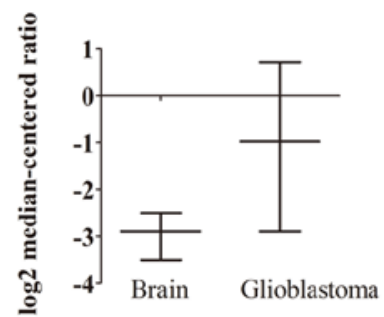

E

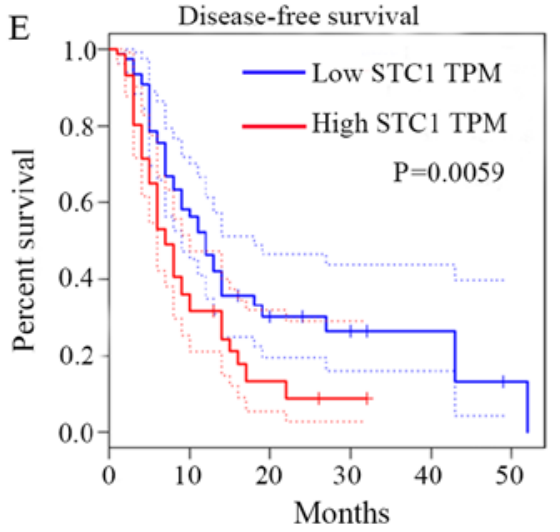

$\mathrm{C}$

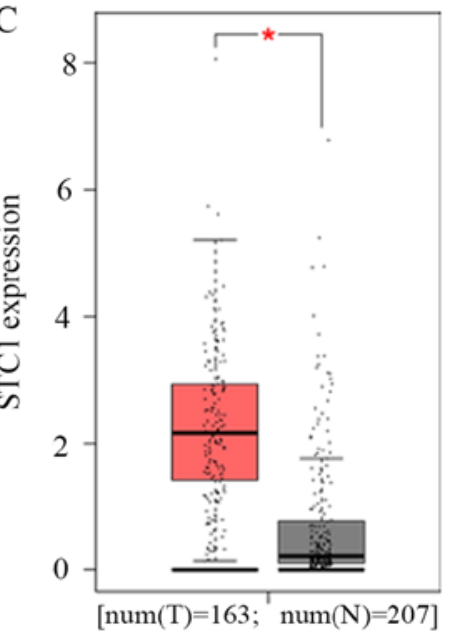

Figure 1. Analysis of the expression levels of STC1. (A) The expression levels of STC1 in Murat brain datasets were analyzed using the Oncomine tool. (B) The expression levels of STC1 in Rickman brain datasets were analyzed using the Oncomine tool. (C) The expression levels of STC1 were analyzed using the GEPIA online tool. Evaluation of the effect of STC1 genes on (D) disease-free survival and (E) overall survival was performed using the GEPIA online tool. Red box represents glioblastoma tissues. Grey box represents normal tissues. STC1, stanniocalcin-1. TPM, transcripts per million.

(1:1,000; catalog no. ab229477), p-SMAD2/3 (1:1,000; catalog no. ab63399), SMAD4 (1:1,000; catalog no. ab191026), Phospho-SMAD4 (1:1,000; catalog no. PA5-64712) and GAPDH (1:1,000; catalog no. ab8245) antibodies (all from Abcam). The secondary antibody (1:5,000; anti-rabbit IgG, HRP-linked; cat. no. 7074) was purchased from Cell Signaling Technology, Inc. SuperBrite ECL western blot substrate and detection kit were purchased from AmyJet Scientific, Inc. The bands were analyzed using Image Lab (version 5.2; Bio-Rad Laboratories, Inc.).

EdU assay. The cellar proliferation ability was detected using an EdU assay, according to the manufacturer's protocol $(24,25)$.

Cell invasion assays. Transwell invasion assays were performed as previously described $(26,27)$. Then, the results were observed with a light microscope (magnification, $\mathrm{x} 20$ ).

Xenograft model. All procedures for the animal experiments were approved by The Committee on The Use and Care of Animals of The Chongqing Red Cross Hospital, and performed in accordance with the institutional guidelines. In total, 20 female, 6-week-old BALB/c nude mice (weight, $16 \mathrm{~g}$ ) were purchased from the Laboratory Animal Center of Chongqing Red Cross Hospital. They were maintained in collective cages in an appropriate room with controlled temperature $\left(22 \pm 2^{\circ} \mathrm{C}\right)$, humidity $(60-70 \%)$ and a 12 -h light/dark cycle, and were fed with standard mouse chow and water. The mice had free access to food and water. The cancer cells were infected with the indicated lentiviral vectors (MOI, 20) and injected $\left(5 \times 10^{6}\right.$ cells/mouse in $\left.100 \mu \mathrm{l}\right)$ subcutaneously into the left armpit. A total of 21 days later, the mice were sacrificed to confirm the presence of tumors and the established tumors were weighed. There was no sign of distress observed in the xenografted mice.

Bioinformatics analysis. Oncomine database (version 3.0; https://www.oncomine.org/) was used to analyze the glioblastoma-derived transcriptomes. The Cancer Genome Atlas (TCGA) (https://www.cancer.gov/about-nci/organization/ccg/research/structural-genomics/tcga) database was also investigated using the GEPIA (version 2) online tool (http://gepia.cancer-pku.cn/). StarBase software (version 2.0; http://starbase.sysu.edu.cn/starbase2/index.php) was used to investigate the interaction networks of different types of RNAs.

Statistical analysis. All statistical analyses were performed using SPSS software (version 19.0; SPSS, Inc.). Each experiment was performed in triplicate. The statistical analysis was performed using a Student's t-test or ANOVA followed by Scheffe's test. Data are presented as the mean \pm SD. $\mathrm{P}<0.05$ was considered to indicate a statistically significant result.

\section{Results}

STC1 is associated with poor outcomes in patients with glioblastoma. In the present study, the expression patterns in glioblastoma were analyzed using the Oncomine database, and the expression levels of STC1 were increased in glioblastoma samples when compared with normal brain tissues in both Murat brain and Rickman brain datasets (28) (Fig. 1A and B). In addition, the expression patterns in glioblastoma were analyzed using TCGA database and GEPIA online tool. The 

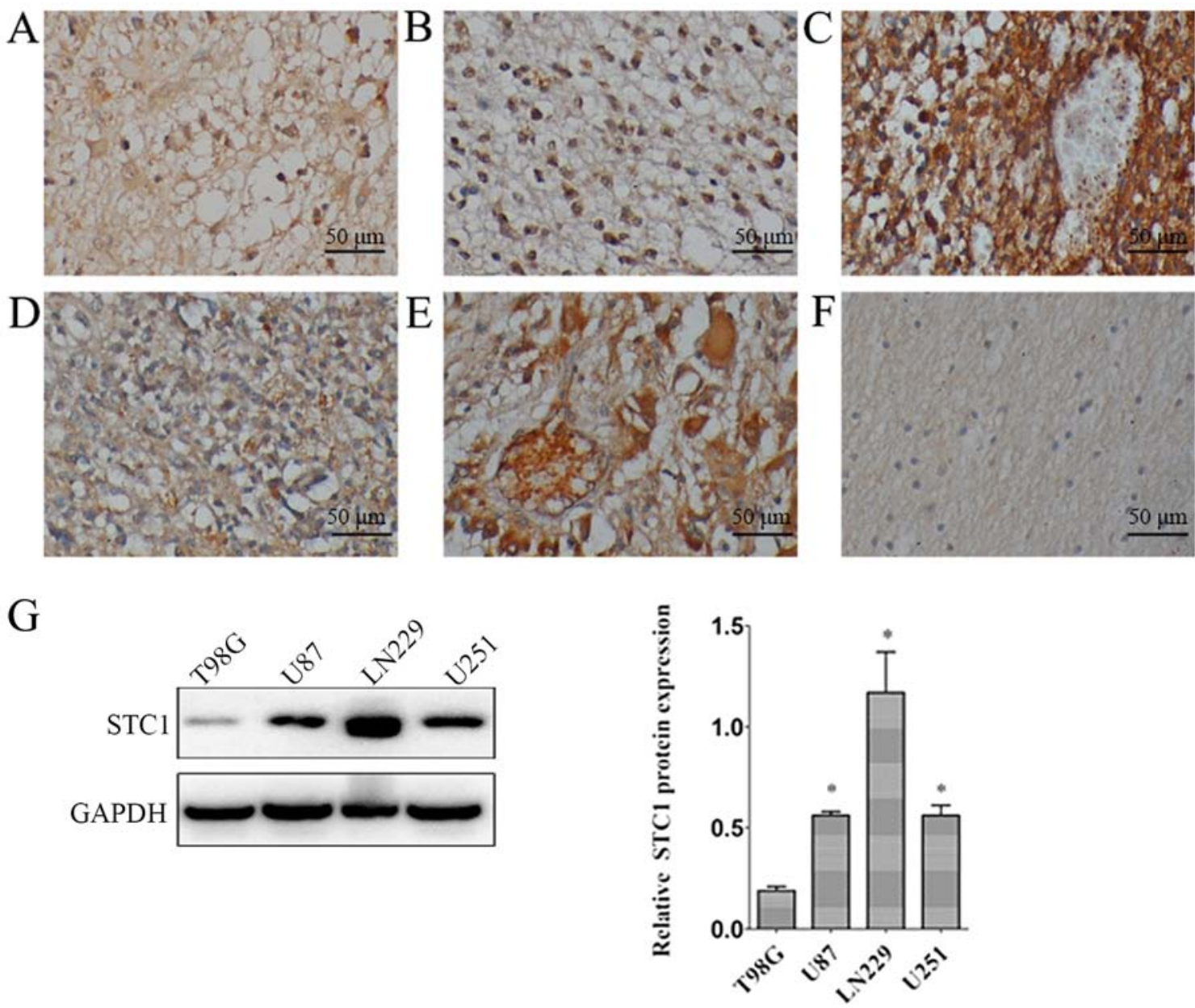

Figure 2. STC1 expression in glioblastoma tissues and cell lines. (A) STC1 staining in Anaplastic oligodendroglioma. (B) STC1 staining in Astrocytoma. (C) STC1 staining in Astrocytoma. (D) STC1 staining in Glioblastoma. (E) STC1 staining in Giant cell glioblastoma. (F) STC1 staining in normal brain samples. (G) The expression levels of STC1 in cell lines were assessed via western blotting. Error bars represent the standard error. " $\mathrm{P}<0.05$ vs. T98G. STC1, stanniocalcin-1.

expression levels of STC1 in glioblastoma were revealed to be higher than those in the normal brain tissues (Fig. 1C). The disease-free survival (DFS) and overall survival (OS) of STC1 genes was evaluated using the GEPIA online tool (http://gepia. cancer-pku.cn/). Low expression levels of STC1 mRNA were significantly associated with the optimal DFS and OS for all patients with glioblastoma (Fig. 1D and E).

Increased expression of STC1 in glioblastoma tissues. The expression patterns of STC1 in glioblastoma and normal brain tissue samples were investigated using IHC. STC1 was primarily localized in the cytoplasm (Fig. 2A-E). The staining intensity was significantly higher in the glioblastoma samples when compared with that in the normal brain samples (Fig. 2A-E and Table I). To analyze the association between STC1 expression and tumor grade, the cancer samples were divided into four groups. The expression of STC1 revealed a positive association with cancer grade $(\mathrm{P}<0.05$; Table I), but not with the age of the patients ( $\mathrm{P}>0.05$; Table I). Furthermore, the STC1 expression levels in the U251, U87 and LN229 cell lines were higher than that in the T98G cell line (Fig. 2G).

STC1 regulates proliferation and invasion in glioblastoma cells. To investigate the role of STC1 in glioblastoma cells,
STC1 expression was silenced in glioblastoma cells (Fig. 3A). Ectopic expression of STC1 led to successful overexpression (Fig. 3A). Furthermore, STC1 silencing inhibited the proliferation and invasion of LN229 cells (Fig. 3B-E), while the ectopic expression promoted the proliferation and invasion of $\mathrm{T} 98 \mathrm{G}$ cells (Fig. 3B-E), thereby indicating that STC1 may promote glioblastoma malignancy.

STC1 regulates transforming growth factor- $\beta($ TGF- $\beta) / S M A D 4$ pathway. To explore the potential mechanism of STC1 in glioblastoma, the association between STC1 and other genes was analyzed using the GEPIA online tool. Consequently, a positive correlation was established between STC1 and TGFB1, as determined by GEPIA online tool (Fig. 4A). In addition, STC1 was a potential competing endogenous (ce)RNA of SMAD4 as assessed by starBase software (Table SI). Thus, STC1 may be ascribed to the regulation of the TGF- $\beta /$ SMAD4 pathway. Silencing STC1 inhibited the expression of p-SMAD2/3, SMAD4 and p-SMAD4 in LN229 cells (Fig. 4B), while the ectopic expression of STC1 promoted the expression of p-SMAD2/3, SMAD4 and p-SMAD4 in T98G cells (Fig. 4B).

STC1 regulates miR-34a in a SMAD4-dependent manner. The present study revealed that STC1 is a potential ceRNA 
Table I. Association between STC1 expression and clinicopathological characteristics in 40 patients with glioblastoma tissues.

STC-1 expression level

Characteristics

Patients, $\mathrm{n}(\mathrm{n}=80)$

Low, n (\%)

High, n (\%)

P-value

Age, years

$<50 \quad 36$

36
44

$16(44.44)$

$20(55.56)$

$>0.05$

$\geq 50$

$21(47.73)$

$23(52.27)$

Tissue type

Normal

40

$30(75.00)$

$10(25.00)$

$<0.05$

Cancerous

40

7 (17.50)

33 (82.50)

Grade

1

8

3 (37.50)

5 (62.50)

2 (22.22)

7 (77.78)

3

9

1 (8.33)

11 (91.67)

4

11

1 (9.09)

$10(90.91)$

a'Grades 3-4 vs. grades 1-2. STC1, stanniocalcin-1.

A

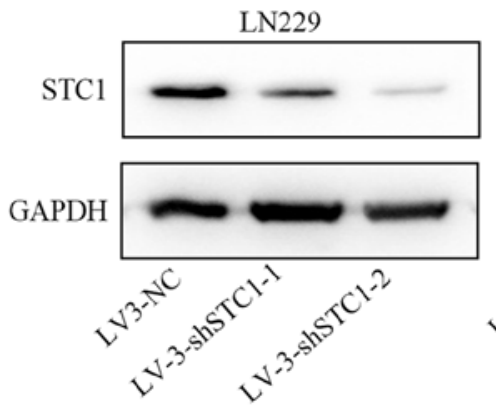

B

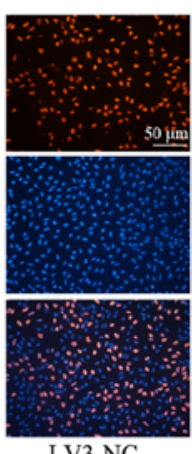

LV3-NC
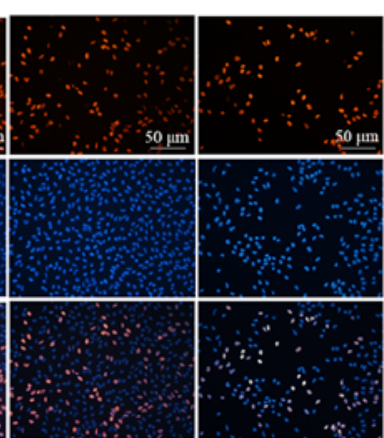

LV3-shSTC1

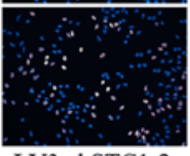

LV3-shSTC1-2

D

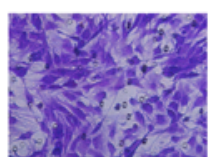

LV3-NC

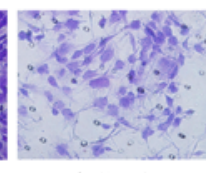

LV3-shSTC1-1 LV3-shSTC1-2
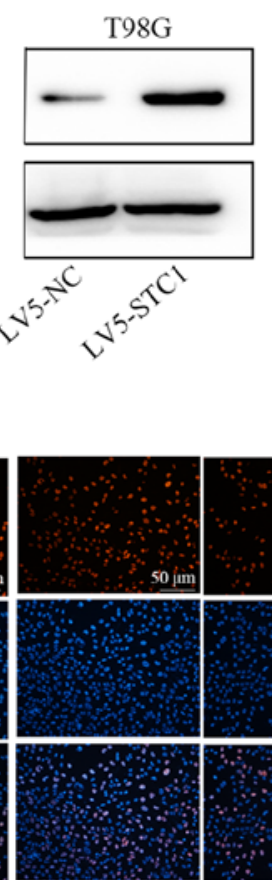

LV5-NC

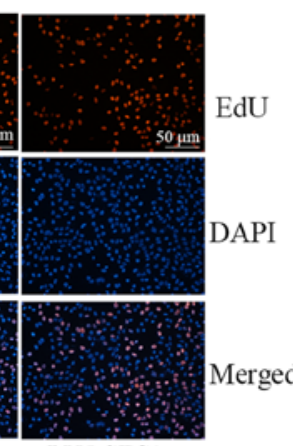

LV5-STC1
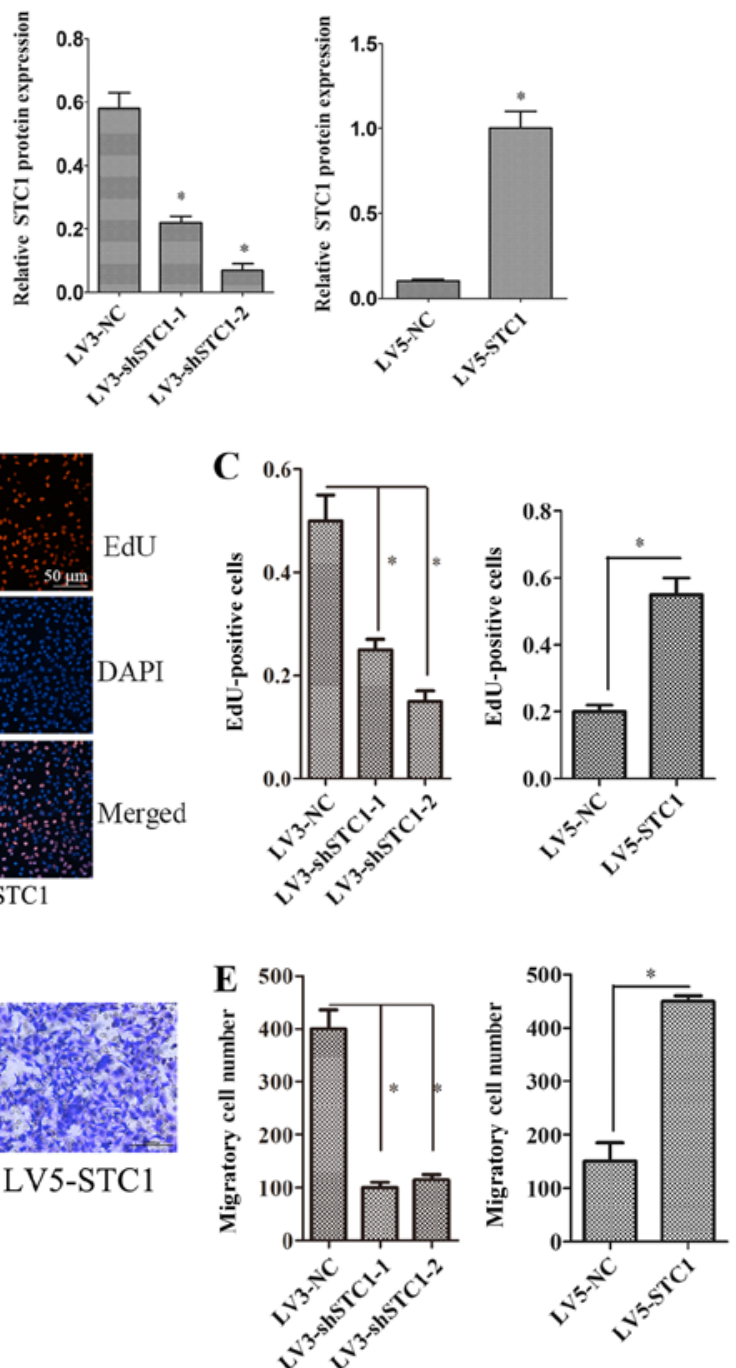

Figure 3. Cellular proliferation and invasion, determined via EdU and Transwell assays. (A) Knockdown of STC1 inhibited the expression level of STC1 in LN229 cells and overexpression of STC1 promoted the expression level of STC1 in T98G cells. (B) Knockdown of STC1 inhibited the proliferation in LN229 cells and overexpression of STC1 promoted proliferation in T98G cells. (C) Quantification of Edu-positive cells. (D) Knockdown of STC1 inhibited the migration of in LN229 cells and Overexpression of STC1 promoted invasion in T98G cells. (E) Quantification of migration assay. Error bars represent the standard error. " $\mathrm{P}<0.05$. STC1, stanniocalcin-1. 
A

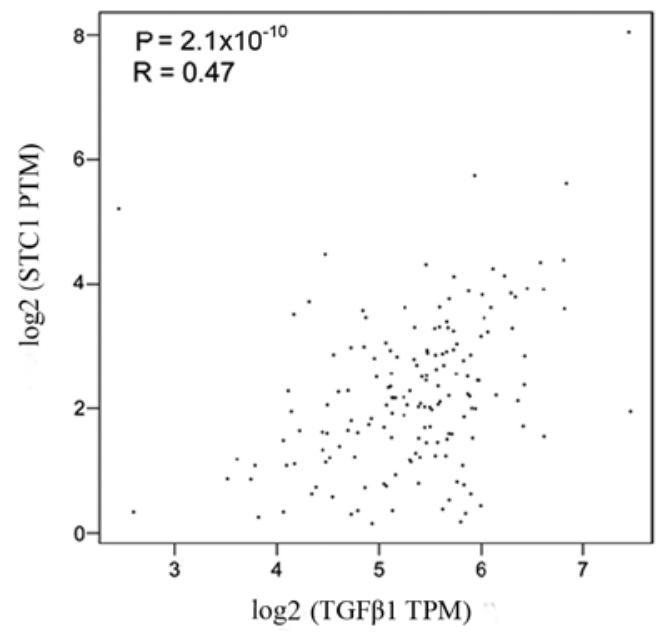

B
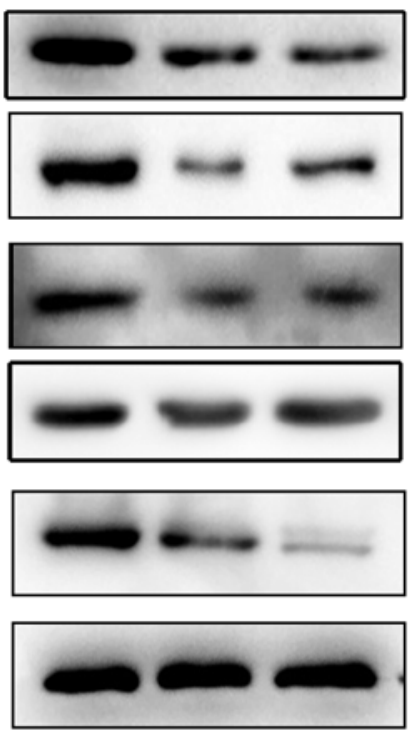

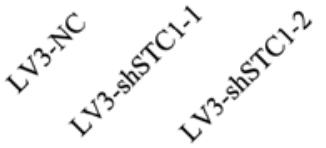
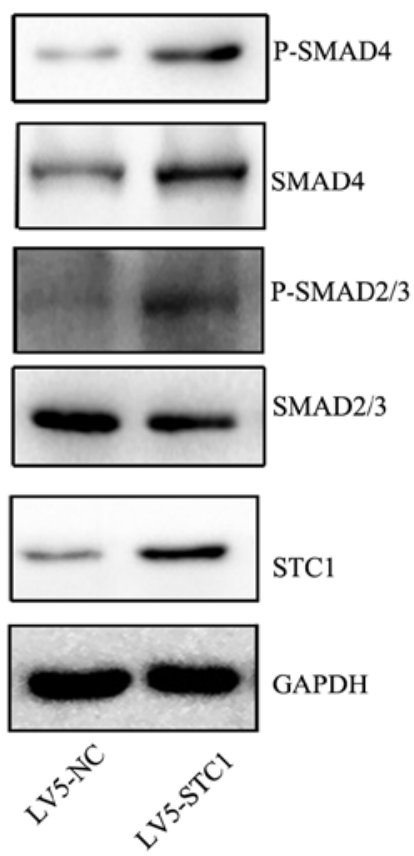
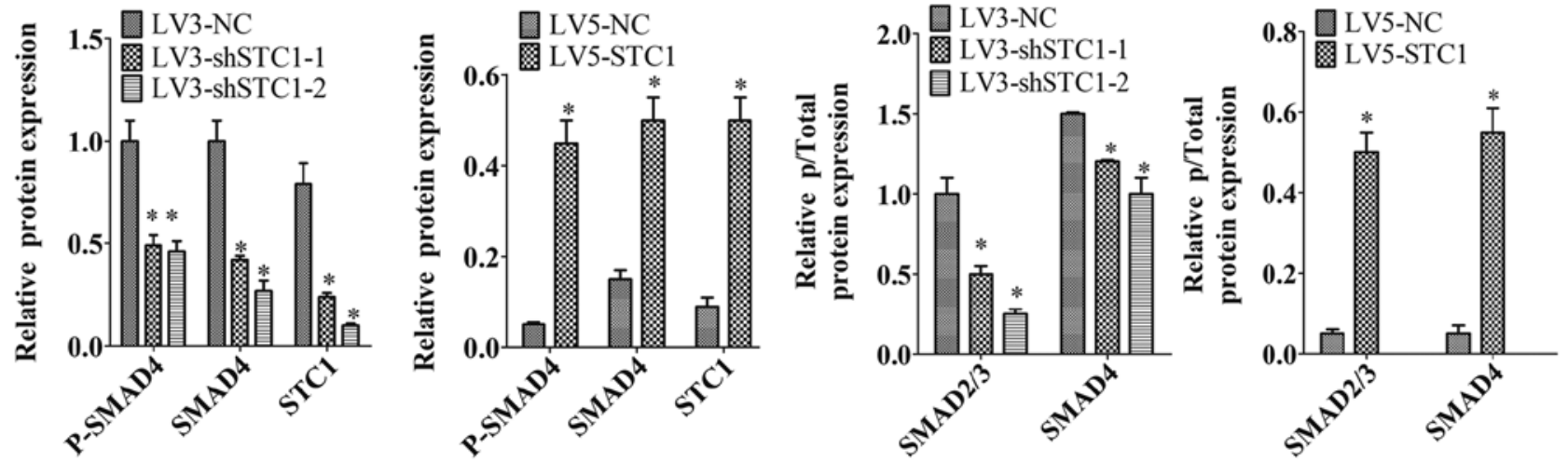

Figure 4. Western blot analysis. (A) The correlation between STC1 and TGF $\beta 1$ was analyzed. (B) The expression levels of STC1, P-SMAD2/3 and SMAD4 were detected via western blotting. *P<0.05 vs. respective LV3-NC group. STC1, stanniocalcin-1; TGF $\beta 1$, tumor growth factor $\beta 1$. TPM, transcripts per million.

of SMAD4 that competes for multiple miRNAs (including miR-130ac, miR-15abc and miR-34ac) (http://starbase.sysu. edu.cn/starbase2/index.php).

It was observed that the expression levels of miR-34a and miR-34c were significantly increased in LN229 cells infected with LV3-shSTC1-1 or LV3-shSTC1-2 when compared with LV3-NC (Fig. 5A). The expression levels of miR-34a and miR-34c were suppressed in T98G cells infected with LV5-STC1 compared with LV5-NC (Fig. 5B). The expression levels of miR-34a were significantly increased when transfected with miR-34 mimics. By contrast, the expression of miR-34a was significantly decreased when transfected with miR-34 inhibitors (Fig. 5C). The expression levels of STC1 and SMAD4 were decreased when transfected with miR-34a mimics in LN229 cells (Fig. 5D). Conversely, the expression of STC1 and SMAD4 was increased when transfected with miR-34a inhibitors (Fig. 5D). In addition, the silencing of STC1 inhibited the expression of SMAD4. However, this regulation was abrogated when the cells were transfected with miR-34a inhibitors (Fig. 5E). The dual-luciferase reporter gene assay confirmed that STC1 and SMAD4 were the direct targets of
miR-34a (Fig. 5F). These data indicated that STC1 regulated miR-34a in a SMAD4-dependent manner.

STC1 inhibited LN229 cells growth in vivo. The role of STC1 in tumor formation of LN229 cells was investigated in an animal model. LV3-shSTC1-1- and LV3-NC-infected LN229 cells formed tumors in all nude mice. The average weight of the tumors was significantly lower in the LV3-shSTC1-1-infected group than that in the LV3-NC-infected group $(\mathrm{P}<0.05$; Fig. 6A and B). The average volume of the tumors in the LV3-shSTC1-1-infected group was significantly lower than that of the LV3-NC-infected group $(\mathrm{P}<0.05$; Fig. $6 \mathrm{~A}$ and $\mathrm{B})$. The expression levels of $\mathrm{p}-\mathrm{SMAD} 2 / 3$, SMAD4 and STC1 were decreased in the LV3-shSTC1-1 group (Fig. 6C). These data suggested that STC1 knockdown may suppress tumor growth.

\section{Discussion}

In the present study, increased STC1 expression levels were observed in glioblastoma tissues. In addition, the silencing of 
A

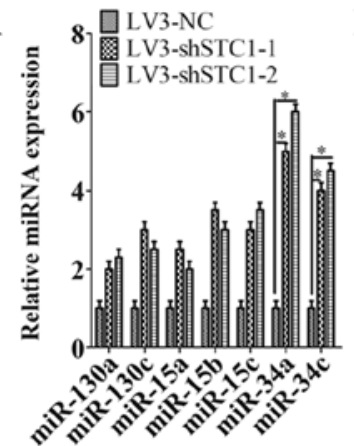

B

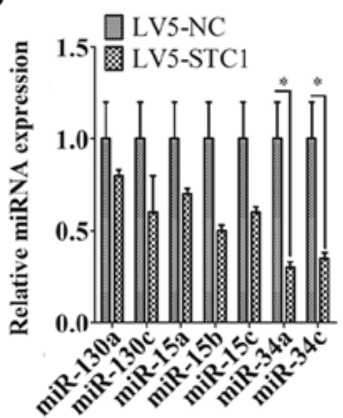

C

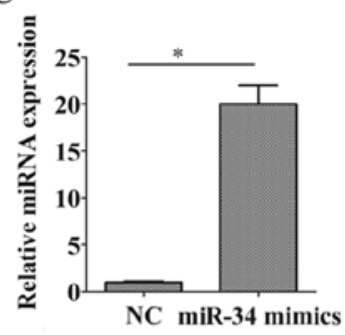

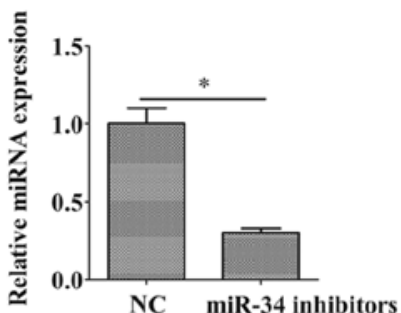

$\mathbf{E}$
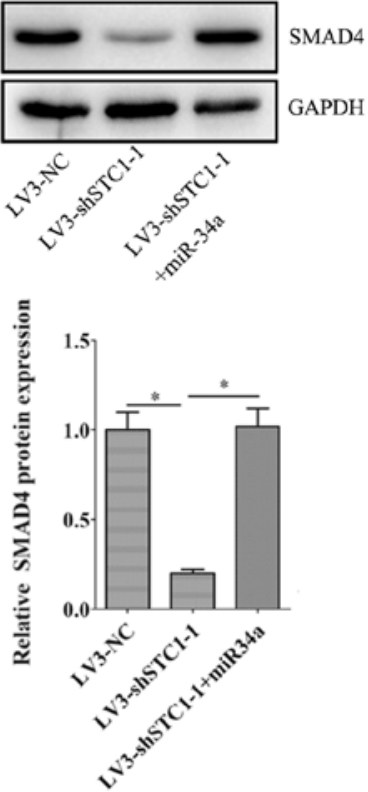

$\mathbf{F}$

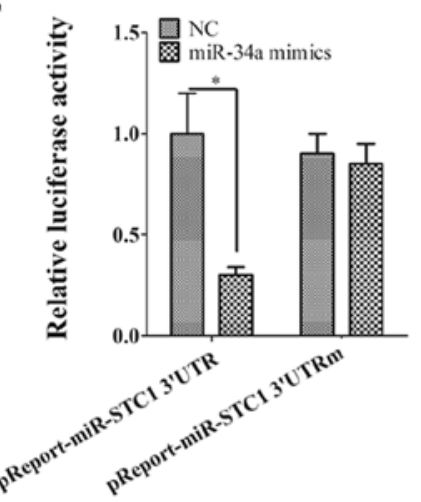

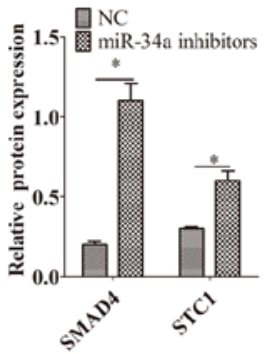

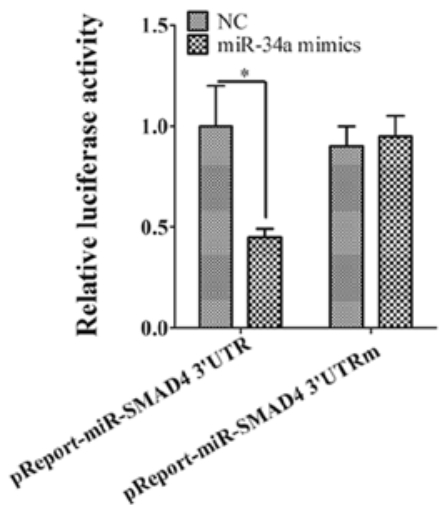

SMAD4 3' UTR 5'AAUAAAUCACUGCC3' (2995-3002)

STC1 3' UTR 5'CUUAUGGACACUGCC3'

(2021-2028)

hsa-miR-34a 3'CGAUUCUGUGACGG5'

Figure 5. miR-34a regulates the expression of SMAD4 and STC1. (A) Silencing STC1 affected the expression levels of various miRNAs in LN229 cells. (B) Overexpression of STC1 regulated the expression level of STC1 in T98G cells. (C) Effect of miR-34a mimics and inhibitors. (D and E) miR-34a regulated the expression of STC1 and SMAD4. (F) A dual luciferase reporter gene assay was performed. Error bars represent the standard error. "P<0.05. miRNA, microRNA; miR, microRNA; STC1, stanniocalcin-1; NC, negative control; 3'UTR, 3' untranslated region.

STC1 inhibited glioblastoma cell malignancy. STC1 is known to regulate the TGF- $\beta /$ SMAD4 pathway. The low expression level of STC1 mRNA was significantly associated with optimal DFS and OS for all patients with glioblastoma. Therefore, STC1 is a potential therapeutic target for glioblastoma and an independent predictor of prognosis.

A number of studies have demonstrated that STC1 plays a major role in cellular proliferation and apoptosis. Another study found that STC1 inhibited cellular apoptosis in colorectal cancer, ovarian cancer, breast cancer, thyroid cancer and glioma tumor $(12,29-31)$. STC1 regulated the proliferation and invasion of cervical cancer cells via the
NF- $\kappa$ B pathway (21). Another study demonstrated that the elevated expression levels of STC-1 promoted the invasion of triple negative breast cancer cells through the activation of the JNK/c-Jun signaling pathway (22). In the present study, increased expression levels of STC1 were revealed in glioblastoma tissues. In addition, silencing of STC1 inhibited glioblastoma malignancy. The data revealed that increased expression levels of STC1 in glioblastoma may promote the progression of glioblastoma.

SMAD4 is a mediator of the TGF- $\beta$ signaling pathway. High expression levels of SMAD4 result in upregulated expression of matrix metalloproteinase 9 and promote the progression of 
A

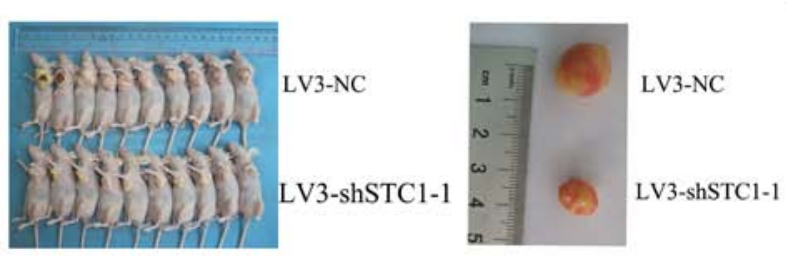

$\mathrm{C}$
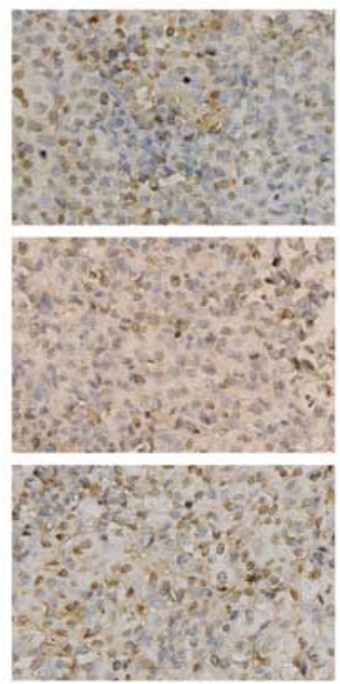

LV3-NC
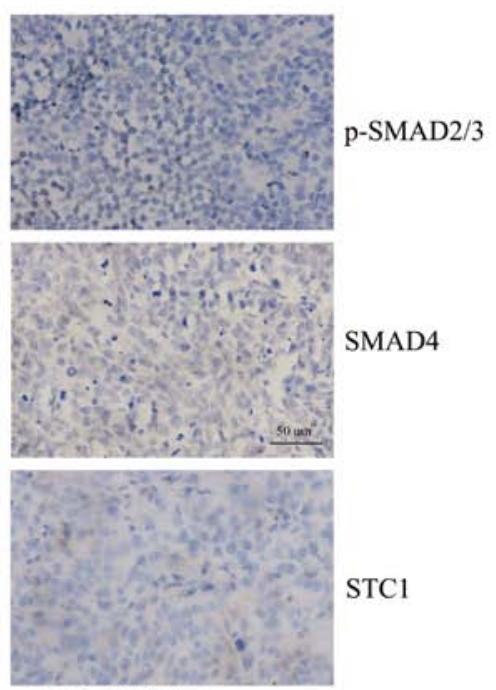

LV3-shSTC1-1

SMAD4

STC1
$\mathrm{B}$
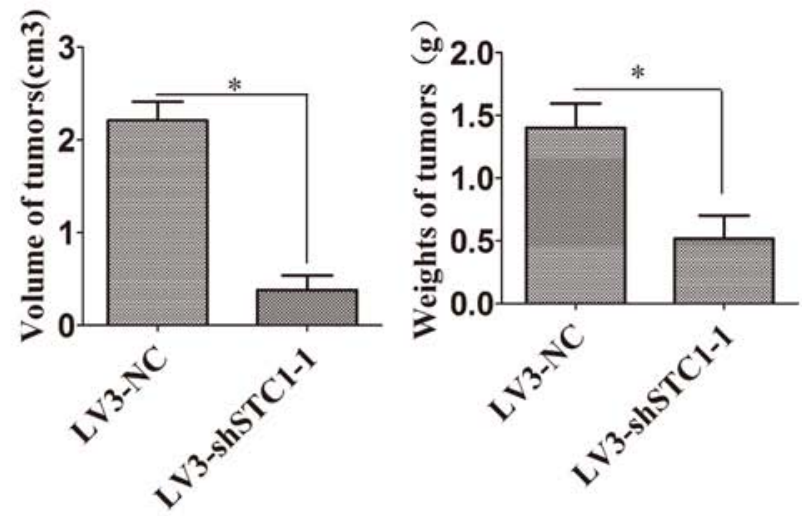

Figure 6. STC1 regulates the expression levels of STC1, p-SMAD2/3 and SMAD4 in vivo. (A and B) Silencing of STC1 inhibited the growth of tumors in vivo. (C) The expression levels of STC1, p-SMAD2/3 and SMAD4 were detected using immunohistochemistry. Error bars represent the standard error. "P<0.05. STC1, stanniocalcin-1.

glioma (32). In C6 glioma cells, miR-124 inhibits proliferation by directly targeting SMAD4 (33). In the present study, it was observed that STC1 regulated the expression of SMAD2/3 and SMAD4, indicating that it may regulate the TGF- $\beta /$ SMAD4 pathway.

By acting as ceRNAs, miRNAs are able to regulate mRNAs, long non-coding (lnc)RNAs and circular RNAs (34). Starbase is a network database used to predict mRNA or lncRNA regulation via miRNA $(35,36)$. In the present study, it was predicted that STC-1 acted as a ceRNA of SMAD4 via miR-34a. This was confirmed when investigations revealed that the mutual regulation between STC1 and SMAD4 was dependent on miR-34a.

miR-34a was associated with the proliferation and apoptosis of meningioma cells. In addition, SMAD4 was identified as a direct target for miR-34a (37). miR-34a represses the endometrial cancer cell invasion and sphere formation (38). miR-34a is also a multi-drug-resistant molecule and a prognostic indicator of breast cancer, which may regulate drug-resistant breast cancer (39). Thus, miR-34a may act as a tumor promoter. In the present study it was observed that STC1 and SMAD2 were direct targets of miR-34a. STC1 regulated SMAD2 through miR-34a. These data indicated that STC1 may promote glioblastoma malignancy. miR-34a was also involved in the TGF- $\beta 1$ pathway. TGF- $\beta 1$ was secreted by tumor-associated macrophages, and promoted colorectal cancer cell proliferation and invasion by regulating the miR-34a/vascular endothelial growth factor axis (40). The present study demonstrated that STC1 regulates the TGF- $\beta / S M A D 4$ pathway. STC1 was a direct target of miR-34a. Thus, the miR-34a/STC1 axis promoted cancer progression via the TGF- $\beta$ /SMAD4 pathway.

In conclusion, aberrant expression levels of STC1 were observed in glioblastoma tissues in the present study. In addition, the silencing of STC1 inhibited glioblastoma malignancy; these biological effects were associated with the TGF- $\beta /$ SMAD4 pathway.

\section{Acknowledgements}

Not applicable.

\section{Funding}

No funding was received.

\section{Availability of data and materials}

The datasets used and/or analyzed during the present study are available from the corresponding author on reasonable request.

\section{Authors' contributions}

YX designed and performed the experiments. QW analyzed the data and wrote the article. 


\section{Ethics approval and consent to participate}

The present study was approved by the Ethics Committee of The Chongqing Red Cross Hospital, and written informed consent was obtained from all patients. All procedures for animal experiments were approved by the Committee on the Use and Care of Animals of The Chongqing Red Cross Hospital, and performed in accordance with the institution guidelines.

\section{Patient consent for publication}

Not applicable.

\section{Competing interests}

The authors declare that they have no competing interests.

\section{References}

1. Millauer B, Shawver LK, Plate KH, Risaui W and Ullrich A: Glioblastoma growth inhibited in vivo by a dominant-negative Flk-1 mutant. Nature 367: 576-579, 1994.

2. Ohgaki $\mathrm{H}$ and Kleihues P: Genetic pathways to primary and secondary glioblastoma. Am J Pathol 170: 1445-1453, 2007.

3. Vredenburgh JJ, Desjardins A, Herndon JE II, Marcello J, Reardon DA, Quinn JA, Rich JN, Sathornsumetee S, Gururangan S, Sampson J, et al: Bevacizumab plus irinotecan in recurrent glioblastoma multiforme. J Clin Oncol 25: 4722-4729, 2007.

4. Wang J, Cazzato E, Ladewig E, Frattini V, Rosenbloom DI, Zairis S, Abate F, Liu Z, Elliott O, Shin YJ, et al: Clonal evolution of glioblastoma under therapy. Nat Genet 48: 768-776, 2016.

5. Brown CE, Alizadeh D, Starr R, Weng L, Wagner JR, Naranjo A, Ostberg JR, Blanchard MS, Kilpatrick J, Simpson J, et al: Regression of glioblastoma after chimeric antigen receptor T-cell therapy. N Engl J Med 375: 2561-2569, 2016.

6. Iwadate Y: Epithelial-mesenchymal transition in glioblastoma progression. Oncol Lett 11: 1615-1620, 2016.

7. Ozeki S, Baba I, Takaya N and Shoun H: A novel C1-using denitrifier alcaligenes sp. STC1 and its genes for copper-containing nitrite reductase and azurin. Biosci Biotechnol Biochem 65: 1206-1210, 2001

8. Wagner GF, Hampong M, Park CM and Copp DH: Purification, characterization, and bioassay of teleocalcin, a glycoprotein from salmon corpuscles of Stannius. Gen Comp Endocrinol 63 : 481-491, 1986.

9. Schein V, Cardoso JC, Pinto PI, Anjos L, Silva N, Power DM and Canário AV: Four stanniocalcin genes in teleost fish: Structure, phylogenetic analysis, tissue distribution and expression during hypercalcemic challenge. Gen Comp Endocrinol 175: 344-356, 2012.

10. Kawabata M, Umemoto N, Shimada Y, Nishimura Y, Zhang B Kuroyanagi J, Miyabe M and Tanaka T: Downregulation of stanniocalcin 1 is responsible for sorafenib-induced cardiotoxicity. Toxicol Sci 143: 374-384, 2015.

11. Ma X, Gu L, Li H, Gao Y, Li X, Shen D, Gong H, Li S, Niu S, Zhang Y, et al: Hypoxia-induced overexpression of stanniocalcin-1 is associated with the metastasis of early stage clear cell renal cell carcinoma. J Transl Med 13: 56, 2015.

12. Su J, Guo B, Zhang T, Wang K, Li X and Liang G: Stanniocalcin-1, a new biomarker of glioma progression, is associated with prognosis of patients. Tumour Biol 36: 6333-6339, 2015.

13. Du YZ, Gu XH, Cheng SF, Li L, Liu H, Hu LP and Gao F: The oncogenetic role of stanniocalcin 1 in lung adenocarcinoma: A promising serum candidate biomarker for tracking lung adenocarcinoma progression. Tumour Biol 37: 5633-5644, 2016.

14. Jepsen MR, Kløverpris S, Bøtkjær JA, Wissing ML, Andersen CY and Oxvig C: The proteolytic activity of pregnancy-associated plasma protein-A is potentially regulated by stanniocalcin- 1 and -2 during human ovarian follicle development. Hum Reprod 31 : 866-874, 2016.
15. Law AY and Wong CK: Stanniocalcin-1 and -2 promote angiogenic sprouting in HUVECs via VEGF/VEGFR2 and angiopoietin signaling pathways. Mol Cell Endocrinol 374: 73-81, 2013.

16. Tang SE, Wu CP, Wu SY, Peng CK, Perng WC, Kang BH, Chu SJ and Huang KL: Stanniocalcin-1 ameliorates lipopolysaccharide-induced pulmonary oxidative stress, inflammation, and apoptosis in mice. Free Radic Biol Med 71: 321-331, 2014

17. Cornmark L, Lønne GK, Jögi A and Larsson C: Protein kinase $\mathrm{C} \alpha$ suppresses the expression of STC1 in MDA-MB-231 breast cancer cells. Tumour Biol 32: 1023-1030, 2011.

18. Tamura S, Oshima T, Yoshihara K, Kanazawa A, Yamada T, Inagaki D, Sato T, Yamamoto N, Shiozawa M, Morinaga S, et al: Clinical significance of STC1 gene expression in patients with colorectal cancer. Anticancer Res 31: 325-329, 2011.

19. Yeung BH, Shek FH, Lee NP and Wong CK: Stanniocalcin-1 reduces tumor size in human hepatocellular carcinoma. PLoS One 10: e0139977, 2015.

20. Leung CC and Wong CK: Effects of STC1 overexpression on tumorigenicity and metabolism of hepatocellular carcinoma. Oncotarget 9: 6852-6861, 2018.

21. Pan X, Jiang B, Liu J, Ding J, Li Y, Sun R, Peng L, Qin C, Fang S and Li G: STC1 promotes cell apoptosis via NF- $\mathrm{kB}$ phospho-P65 Ser536 in cervical cancer cells. Oncotarget 8: 46249-46261, 2017.

22. Han J, Jeon M, Shin I and Kim S: Elevated STC-1 augments the invasiveness of triple-negative breast cancer cells through activation of the JNK/c-Jun signaling pathway. Oncol Rep 36: 1764-1771, 2016.

23. Hu J, Meng Y, Zhang Z, Yan Q, Jiang X, Lv Z and Hu L: MARCH5 RNA promotes autophagy, migration, and invasion of ovarian cancer cells. Autophagy 13: 333-344, 2017.

24. Kurio N, Saunders C, Bechtold TE, Salhab I, Nah HD, Sinha S, Billings PC, Pacifici M and Koyama E: Roles of Ihh signaling in chondroprogenitor function in postnatal condylar cartilage. Matrix Biol 67: 15-31, 2018.

25. Tao L, Bei Y, Li Y and Xiao J: Neonatal rat cardiomyocytes isolation, culture, and determination of microRNAs' effects in proliferation. Methods Mol Biol 1733: 203-213, 2018.

26. Min X, Liu K, Zhu $\mathrm{H}$ and Zhang J: Long noncoding RNA LINC003121 inhibits proliferation and invasion of thyroid cancer cells by suppression of the phosphatidylinositol-3-kinase (PI3K)/Akt signaling pathway. Med Sci Monit 24: 4592-4601, 2018.

27. Yang Z, Li K, Liang Q, Zheng G, Zhang S, Lao X, Liang Y and Liao G: Elevated hydrostatic pressure promotes ameloblastoma cell invasion through up-regulation of MMP-2 and MMP-9 expression via $W n t / \beta$-catenin signalling. J Oral Pathol Med 47: 836-846, 2018.

28. Pan Y, Yuan F, Li Y, Wang G, Lin Z and Chen L: Bromodomain PHD-finger transcription factor promotes glioma progression and indicates poor prognosis. Oncol Rep 41: 246-256, 2019.

29. Yeung HY, Lai KP, Chan HY, Mak NK, Wagner GF and Wong CK: Hypoxia-inducible factor-1-mediated activation of stanniocalcin-1 in human cancer cells. Endocrinology 146: 4951-4960, 2005

30. Peña C, Céspedes MV, Lindh MB, Kiflemariam S, Mezheyeuski A, Edqvist PH, Hägglöf C, Birgisson H, Bojmar L, Jirström K, et al: STC1 expression by cancer-associated fibroblasts drives metastasis of colorectal cancer. Cancer Res 73: 1287-1297, 2013.

31. Chang AC, Doherty J, Huschtscha LI, Redvers R, Restall C, Reddel RR and Anderson RL: STC1 expression is associated with tumor growth and metastasis in breast cancer. Clin Exp Metastasis 32: 15-27, 2015.

32. Karthikeyan A, Gupta N, Tang C, Mallilankaraman K, Silambarasan M, Shi M, Lu L, Ang BT, Ling EA and Dheen ST: Microglial SMAD4 regulated by microRNA-146a promotes migration of microglia which support tumor progression in a glioma environment. Oncotarget 9: 24950-24969, 2018.

33. Zhang Z, Gong Q, Li M, Xu J, Zheng Y, Ge P and Chi G: MicroRNA-124 inhibits the proliferation of C6 glioma cells by targeting Smad4. Int J Mol Med 40: 1226-1234, 2017.

34. Liu H, Xu D, Zhong X, Xu D, Chen G, Ge J and Li H: LncRNA-mRNA competing endogenous RNA network depicts transcriptional regulation in ischaemia reperfusion injury. J Cell Mol Med 23: 2272-2276, 2019.

35. Chen HH,Zong J and Wang SJ: LncRNA GAPLINC promotes the growth and metastasis of glioblastoma by sponging miR-331-3p. Eur Rev Med Pharmacol Sci 23: 262-270, 2019.

36. Chen J, Du G, Chang Y, Wang Y, Shi L, Mi J and Tang G: Downregulated miR-27b promotes keratinocyte proliferation by targeting PLK2 in oral lichen planus. J Oral Pathol Med 48: 326-334, 2019. 
37. Werner TV, Hart M, Nickels R, Kim YJ, Menger MD, Bohle RM, Keller A, Ludwig N and Meese E: MiR-34a-3p alters proliferation and apoptosis of meningioma cells in vitro and is directly targeting SMAD4, FRAT1 and BCL2. Aging (Albany NY) 9: 932-954, 2017.

38. Dong P, Xiong Y, Yue J, Hanley SJB and Watari H: miR-34a, miR-424 and miR-513 inhibit MMSET expression to repress endometrial cancer cell invasion and sphere formation. Oncotarget 9: 23253-23263, 2018

39. Li ZH, Weng X, Xiong QY, Tu JH, Xiao A, Qiu W, Gong Y, Hu EW, Huang S and Cao YL: miR-34a expression in human breast cancer is associated with drug resistance. Oncotarget 8: 106270-106282, 2017
40. Zhang D, Qiu X, Li J,Zheng S, Li L and Zhao H: TGF- $\beta$ secreted by tumor-associated macrophages promotes proliferation and invasion of colorectal cancer via miR-34a-VEGF axis. Cell Cycle 17: 2766-2778, 2018.

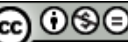

This work is licensed under a Creative Commons Attribution-NonCommercial-NoDerivatives 4.0 International (CC BY-NC-ND 4.0) License. 